PROCEEDINGS OF THE AMERICAN MATHEMATICAL SOCIETY

Volume 135, Number 4, April 2007, Pages 1051-1058

S 0002-9939(06)08684-9

Article electronically published on September 18, 2006

\title{
EQUIVALENCE OF COMPLETENESS AND CONTRACTION PROPERTY
}

\author{
SHU-WEN XIANG
}

(Communicated by Jonathan M. Borwein)

\begin{abstract}
In this paper, we consider the completeness and the contraction property in metric spaces and show that the contraction property implies Lipschitz-completeness or arcwise-completeness in a metric space. However, in a metric space, the contraction property does not imply the usual completeness. We prove that a locally Lipschitz-connected metric space has the contraction property if and only if it is Lipschitz-complete and that a locally arcwise-connected metric space is arcwise-complete if and only if $X$ has the strong contraction property.
\end{abstract}

\section{INTRODUCTION}

Many authors have considered the topological characterization and the equivalence of the contraction property (see [1]-[6], 8]). In [7, Kirk showed that the Caristi fixed point theorem holds only in a complete metric set and Sullivan [9] showed the same for the Ekeland principle. These lead naturally to the question whether a metric space with the contraction property is complete.

Borwein 1 proved the interesting result that a uniformly Lipschitz-connected subset (such as a convex subset of a normed space) has the contraction property if and only if the subset is complete. This implies that a normed space is complete if and only if every contraction on the space has a fixed point. In [1], Borwein also showed that one cannot hope to extend this result much, as the following examples illustrate (see, Examples 3, 4 in [1]).

Example 1.1. There exists an incomplete nonuniformly Lipschitz-connected subset of the Euclidean plane with the contraction property. Let

$$
C=\{(x, y) \mid 0<x \leq 1, y=\sin (1 / x)\} .
$$

Example 1.2. There exists an incomplete starshaped subset of the Euclidean plane with the contraction property. Let

$$
C=\bigcup\left\{L_{k} \mid k \in N\right\},
$$

where

$$
L_{k}=\operatorname{conv}\left\{(0,0),\left(1,2^{-k}\right)\right\} .
$$

Received by the editors October 22, 2005.

2000 Mathematics Subject Classification. Primary 47H10, 54H25; Secondary 54E50, 54E35.

Key words and phrases. Completeness, contraction property, Lipschitz-completeness, arcwisecompleteness.

This work was completed with the support of NSF of China (No: 10561003).

(C)2006 American Mathematical Society Reverts to public domain 28 years from publication 
In fact, Examples 1.1, 1.2 show that the sufficient and necessary condition fails if "uniformly Lipschitz-connected" is replaced by "Lipschitz-connected" or "starshaped". Thus, in a metric space, we are led to ask what completeness the contraction property can guarantee and under what conditions it implies that the contraction property is equivalent to completeness.

In this paper, based on Lipschitz-completeness and arcwise-completeness, we show that a metric space has the contraction property only if the space is Lipschitzcomplete and the converse remains true in Lipschitz-connected spaces. Our proof of Lipschitz-completeness of the space depends on the technique used by Borwein [1] to construct a contraction map. Furthermore, in the sense of equivalent metric, we prove that an arcwise-connected metric space has the strong contraction property if and only if the space is arcwise-complete.

\section{Preliminaries}

Let $(X, d)$ be a metric space. $X$ is said to be of the contraction property if and only if for any selfmap $T$ on $X, T$ has a fixed point whenever $T$ is a Banach contraction under the metric $d$. $X$ is said to be of the strong contraction property if and only if for any selfmap $T$ on $X, T$ has a fixed point in $X$ whenever $T$ is a Banach contraction under some metric $d^{*}$ uniformly equivalent to $d$.

Let us say that a subset $C$ of the metric space $X$ endowed with a metric $d$ is uniformly Lipschitz-connected if there exists a positive constant $L$ such that given any $x^{0}$ and $x^{1}$ in $C$ there exists an arc $g:[0,1] \mapsto C$ with $g(0)=x^{0}$ and $g(1)=x^{1}$ such that

$$
d(g(s), g(t)) \leq L|s-t| d(g(0), g(1))
$$

for $0 \leq s, t \leq 1$ (see [1]). An arc $g:[0,1] \rightarrow C$ with $g(0)=x^{0}$ and $g(1)=x^{1}$ is called a Lipschitz arc if $g$ satisfies the Lipschitz condition, i.e., there exists $L>0$ such that

$$
d(g(s), g(t)) \leq L|s-t|
$$

for $0 \leq s, t \leq 1$. A subset $C$ of $X$ is said to be Lipschitz-connected if for arbitrary $x^{0}$ and $x^{1}$ in $C$ there exists a Lipschitz arc $g:[0,1] \rightarrow C$ such that $g(0)=x^{0}$ and $g(1)=x^{1}$. It is easy to see that a uniformly Lipschitz-connected space is Lipschitz-connected but the converse is not true (see [1]). A subset $C$ of $X$ is said to be locally arcwise-connected (respectively, locally Lipschitz-connected) if there is some $\delta>0$ such that for any $x^{0}$ and $x^{1}$ in $C$ there exists an arc (respectively, a Lipschitz arc) $g$ linking $x^{0}$ and $x^{1}$ whenever $d\left(x_{0}, x_{1}\right)<\delta$.

Lemma 2.1. Let $g_{1}, g_{2}:[0,1] \rightarrow C$ be Lipschitz arcs with $g_{1}(1)=g_{2}(0)$. Then

$$
g(t)= \begin{cases}g_{1}(2 t), & 0 \leq t<\frac{1}{2} \\ g_{2}(2 t-1), & \frac{1}{2} \leq t \leq 1\end{cases}
$$

is also a Lipschitz arc.

Proof. Let $s, t \in[0,1]$. For $s, t \in\left[0, \frac{1}{2}\right]$ there exists a constant $L_{1}>0$ such that

$$
d(g(s), g(t))=d\left(g_{1}(2 s), g_{1}(2 t)\right) \leq L_{1}|s-t|,
$$

and for $s, t \in\left[\frac{1}{2}, 1\right]$, there exists a constant $L_{2}>0$ such that

$$
d(g(s), g(t))=d\left(g_{2}(2 s), g_{2}(2 t)\right) \leq L_{2}|s-t| .
$$


For $s \in\left[0, \frac{1}{2}\right]$ and $t \in\left[\frac{1}{2}, 1\right]$, we have

$$
\begin{aligned}
d(g(s), g(t)) & \leq d\left(g(s), g\left(\frac{1}{2}\right)\right)+d\left(g\left(\frac{1}{2}\right), g(t)\right) \leq L_{1}\left|s-\frac{1}{2}\right|+L_{2}\left|\frac{1}{2}-t\right| \\
& \leq L_{1}\left(\frac{1}{2}-s\right)+L_{2}\left(t-\frac{1}{2}\right) \leq L_{1}(t-s)+L_{2}(t-s) \\
& =\left(L_{1}+L_{2}\right)|t-s| .
\end{aligned}
$$

This implies $g$ is a Lipschitz arc.

Theorem 2.2. Let $C=\bigcup\left\{L_{\alpha}: \alpha \in I\right\}$, where $\left\{L_{\alpha}: \alpha \in I\right\}$ is a family of Lipschitzconnected subsets of a metric space. For arbitrary $L_{\beta}, L_{\gamma} \in\left\{L_{\alpha}: \alpha \in I\right\}$, there exist $L_{\beta}=L_{0}, L_{1}, \cdots, L_{k}, L_{k+1}=L_{\gamma}$ such that $L_{i} \cap L_{i+1} \neq \emptyset$ for all $i=0,1, \cdots, k$. Then $C$ is Lipschitz-connected.

Proof. For $x_{0}, x_{1} \in C$, assume $x_{0} \in L_{\beta}, x_{1} \in L_{\gamma}$. Then there exist $L_{\beta}=L_{0}, L_{1}, \cdots$, $L_{k}, L_{k+1}=L_{\gamma}$ such that $L_{i} \cap L_{i+1} \neq \emptyset$. Since $L_{0}, L_{1}$ are Lipschitz-connected and $L_{0} \cap L_{1} \neq \emptyset$, it is clear from Lemma 2.1 that $L_{0} \cup L_{1}$ is Lipschitz-connected. By Lemma 2.1 and induction, it follows that $\bigcup_{i=0}^{k+1} L_{i}$ is also Lipschitz-connected and there is a Lipschitz arc in $C$ linking $x_{0}$ and $x_{1}$. Hence $C$ is Lipschitz-connected.

In particular $C=\bigcup\left\{L_{\alpha}: \alpha \in I\right\}$ is Lipschitz-connected whenever $\left\{L_{\alpha}: \alpha \in I\right\}$ is a family of Lipschitz-connected subsets of a metric space whose intersection is nonempty. Therefore a starshaped subset of a normed space is Lipschitz-connected. It is easy to check that the subsets of Examples 1.1, 1.2 are Lipschitz-connected. The following example is also a Lipschitz-connected subset.

Example 2.3. Let $C$ be a subset of the Euclidean plane defined by

$$
C=\{(t, 0): t \in[0,1]\} \cup\left(\bigcup_{k=0}^{\infty}\left\{\left(2^{-k}, t\right): t \in[0,1]\right\}\right) .
$$

By Theorem 2.2, $C$ is Lipschitz-connected.

A continuous mapping $g:(0,1] \rightarrow C$ is called a semi-closed arc if for each $\epsilon>0$, there exists some $\delta>0$ such that $d(g(s), g(t))<\epsilon$ for all $0 \leq s, t<\delta$. A semi-closed arc $g$ is called a Lipschitz semi-closed arc if $g$ satisfies the Lipschitz condition.

Definition 2.4. Let $X$ be a metric space.

(1) $X$ is said to be arcwise-complete if for each semi-closed $\operatorname{arc} g:(0,1] \rightarrow X$, $\lim _{s \rightarrow 0} g(s)$ exists in $X$

(2) $X$ is said to be Lipschitz-complete if for each Lipschitz semi-closed arc $g:(0,1] \rightarrow X, \lim _{s \rightarrow 0} g(s)$ exists in $X$.

Remark 2.1. Arcwise-completeness is weaker than usual completeness even if in an arcwise connected space, so is Lipschitz-completeness (see Examples 1.1, 1.2 and 2.3). It is obvious from the definitions that Lipschitz-completeness is weaker than arcwise-completeness.

It is well known that $X$ has usual completeness if and only if $\bigcap F_{n} \neq \emptyset$ whenever $\left\{F_{n}: n \in N\right\}$ is a sequence of nonempty closed subsets of $X$ with $F_{n+1} \subset F_{n}$ and $\operatorname{diam}\left(F_{n}\right) \rightarrow 0$. Concerning Lipschitz-completeness and arcwise-completeness, we have the following properties. 
Theorem 2.5. Let $X$ be a metric space.

(1) $X$ is arcwise-complete if and only if $\bigcap F_{n} \neq \emptyset$ whenever $\left\{F_{n}: n \in N\right\}$ is a sequence of arcwise-connected and nonempty closed subsets of $X$ with $F_{n+1} \subset F_{n}$ and $\operatorname{diam}\left(F_{n}\right) \rightarrow 0$, where $\operatorname{diam}\left(F_{n}\right):=\sup _{x, y \in F_{n}} d(x, y)$ denotes the diameter of $F_{n}$.

(2) $X$ is Lipschitz-complete if and only if $\bigcap F_{n} \neq \emptyset$ whenever $\left\{F_{n}: n \in N\right\}$ is a sequence of Lipschitz-connected and nonempty closed subsets of $X$ with $F_{n+1} \subset F_{n}$ and $\operatorname{diam}\left(F_{n}\right) \rightarrow 0$.

Proof. (i) " $\Rightarrow$ ": Let $X$ be an arcwise-complete space. $\left\{F_{n}: n \in N\right\}$ is a sequence of arcwise-connected and nonempty closed subsets of $X$ with $F_{n+1} \subset F_{n}$ and $\operatorname{diam}\left(F_{n}\right) \rightarrow 0$. For each $F_{n}$, choose $x_{n} \in F_{n}$ and an arc $g_{n}:[0,1] \mapsto F_{n}$ such that $g_{n}(0)=x_{n+1}, g_{n}(1)=x_{n}$. Define $g:(0,1] \mapsto X$ as follows:

$$
g(s)=g_{n}\left(2^{n+1} s-1\right), \quad \forall \frac{1}{2^{n+1}}<s \leq \frac{1}{2^{n}}, \quad n=1,2, \cdots .
$$

This implies from $\operatorname{diam}\left(F_{n}\right) \rightarrow 0$ that $g$ is a semi-closed arc in $X$. The arcwisecompleteness of $X$ implies $\lim _{s \rightarrow 0} g(s)=\bar{x} \in X$. For each $n \in N$, since $F_{n}$ is closed and $g(s) \in F_{n}$ for all $s \in\left(0, \frac{1}{2^{n}}\right], \bar{x} \in F_{n}$, say $\bigcap F_{n} \neq \emptyset$.

" $\Leftarrow$ ": Let $\bar{X}$ be the completion of $X$. Suppose that $X$ were not arcwise-complete. Then there exists some semi-closed arc $g$ in $X$ such that $\lim _{s \rightarrow 0} g(s)=\bar{x} \notin X$. Define $F_{n}$ by

$$
F_{n}=\left\{g(s): s \in\left(0, \frac{1}{2^{n}}\right]\right\}, \quad n \in N .
$$

It is easy to check that $\left\{F_{n}: n \in N\right\}$ is a sequence of arcwise-connected and nonempty closed subsets of $X$ with $F_{n+1} \subset F_{n}$ and $\operatorname{diam}\left(F_{n}\right) \rightarrow 0$. So $\bigcap F_{n} \neq \emptyset$, say $\bar{x} \in F_{n} \subset X$, which is a contradiction.

(ii) Similar to the proof of (i), we only need to replace "arcwise-connected" by "Lipschitz-connected".

\section{THE CONTRACTION PROPERTY AND LIPSCHITZ-COMPLETENESS}

Theorem 3.1. Let $X$ be a metric space. If $X$ has the contraction property, then $X$ is Lipschitz-complete.

Proof. Let $\bar{X}$ be the completion of $X$ and $g:(0,1] \rightarrow X$ be a Lipschitz semi-closed arc. Then

$$
d(g(s), g(t)) \leq L|s-t|, \quad \forall 0<s, t \leq 1 .
$$

Set $\lim _{s \rightarrow 0} g(s)=\bar{x} \in \bar{X}$ and let $h: X \rightarrow[0,1]$ be given by

$$
h(x)=\frac{d(x, \bar{x})}{2 L(1+d(x, \bar{x}))} .
$$

Define $g(0)=\bar{x} \in \bar{X}$ and $X^{\prime}=X \cup\{\bar{x}\}$. Then $g$ extends to a mapping satisfying (3.1) on $[0,1]$. Define $T: X^{\prime} \rightarrow X^{\prime}$ by

$$
T=g \circ h .
$$


$T$ is a metric contraction (with contraction constant $\frac{1}{2}$ ) since

$$
\begin{aligned}
d(T x, T y) & =d\left(g\left(\frac{d(x, \bar{x})}{2 L(1+d(x, \bar{x}))}\right), g\left(\frac{d(y, \bar{x})}{2 L(1+d(y, \bar{x}))}\right)\right) \\
& \leq L\left|\frac{d(x, \bar{x})}{2 L(1+d(x, \bar{x}))}-\frac{d(y, \bar{x})}{2 L(1+d(y, \bar{x}))}\right| \\
& \leq \frac{1}{2}|d(x, \bar{x})-d(y, \bar{x})| \\
& \leq \frac{1}{2} d(x, y) .
\end{aligned}
$$

Note that

$$
\bar{x}=g(0)=g(h(\bar{x}))=T(\bar{x}) .
$$

$\bar{x}$ is the unique fixed point of $T$ in $X^{\prime}$. Finally observing that $T: X \rightarrow X$ and $T$ is also a metric contraction on $X$, we have that the unique fixed point $\bar{x}$ of $T$ is in $X$ and this completes the proof.

In [1, Borwein remarked with some examples that the contraction property holds in some incomplete metric spaces (see Examples 1.1, 1.2). Theorem 3.1 shows that the contraction property cannot ensure usual completeness but can ensure Lipschitz-completeness.

Theorem 3.2. A locally Lipschitz-connected metric space has the contraction property if and only if it is Lipschitz-complete.

Proof. From Theorem 3.1, it remains to show that if $X$ is Lipschitz-complete, then $X$ has the contraction property. Let $T: X \rightarrow X$ be a metric contraction with contraction constant $0 \leq h<1$. Note that $T$ is contractive. Select $x_{0}$ in $X$ with $d\left(x_{0}, T x_{0}\right)<\delta$ and arc $g_{0}:[0,1] \rightarrow X$ which connect $x_{0}$ and $T x_{0}$ in Lipschitz fashion as in (1). For each $k \in N$, define $g_{k}:[0,1] \rightarrow X$ by $g_{k}(s)=T^{k} g_{0}(s)$ for all $s \in[0,1]$. Then $g_{k}$ connects $x_{k}=T^{k}\left(x_{0}\right)$ and $x_{k+1}=T^{k+1}\left(x_{0}\right)$ for each $k \in N$. Define $g:(0,1] \rightarrow X$ by

$$
g(s)=g_{k}(k(k+1) t-k), \quad \frac{1}{k+1}<t \leq \frac{1}{k}, \quad k=1,2, \cdots .
$$

On each interval of the form $\left(\frac{1}{k+1}, \frac{1}{k}\right]$ we have

$$
\begin{aligned}
d(g(s), g(t)) & =\left(g_{k}(k(k+1) s-k), g_{k}(k(k+1) t-k)\right) \\
& =d\left(T^{k} g_{0}(k(k+1) s-k), T^{k} g_{0}(k(k+1) t-k)\right) \\
& \leq h^{k} d\left(g_{0}(k(k+1) s-k), g_{0}(k(k+1) t-k)\right) \\
& \leq L k(k+1) h^{k}|s-t| .
\end{aligned}
$$

Since $0<h<1$ and $\lim _{k \rightarrow \infty} k(k+1) h^{k}=0$, there exists some $N_{0}>0$ such that $k(k+1) h^{k} \leq 1$ for all $k>N_{0}$. Let $M_{1}=\max \left\{1 \cdot 2 h, 2 \cdot 3 h^{2}, \cdots, N_{0}\left(N_{0}+1\right) h^{N_{0}}\right\}$ and $L_{1}=\max \left\{L M_{1}, L\right\}$. Then for each $k \in N$, we have

$$
d(g(s), g(t)) \leq L_{1}|s-t|, \quad \forall s, t \in\left(\frac{1}{k+1}, \frac{1}{k}\right) .
$$


Let $s \in\left(\frac{1}{k+1}, \frac{1}{k}\right), t \in\left(\frac{1}{k+l+1}, \frac{1}{k+l}\right)(k, l \in N)$. Thus by (3.2) and the continuity of $g$, we have

$$
\begin{aligned}
d(g(s), g(t)) & \leq d\left(g(s), g\left(\frac{1}{k+1}\right)\right)+d\left(g\left(\frac{1}{k+1}\right), g\left(\frac{1}{k+2}\right)\right)+\cdots+d\left(g\left(\frac{1}{k+l}\right), g(t)\right) \\
& \leq L_{1}\left|s-\frac{1}{k+l}\right|+L_{1}\left|\frac{1}{k+l}-\frac{1}{k+2}\right|+\cdots+L_{1}\left|\frac{1}{k+l}-t\right| \\
& \leq L_{1}|s-t| .
\end{aligned}
$$

From (3.2) and (3.3), $g:(0,1] \rightarrow X$ is a Lipschitz semi-closed arc in $X$. Then $\bar{x}=\lim _{s \rightarrow 0} g(s) \in X$. Finally observing that $T$ is metric contraction and that $g$ connects $\left\{x^{k}=T^{k} x_{0}: k \in N\right\}$, we have that $\bar{x} \in X$ is a fixed point of $T$.

Corollary 3.3. A starshaped subset of a normed space has the contraction property if and only if it is Lipschitz-complete.

By Theorem 3.2 and Corollary 3.3, we know that the subsets of Examples 1.1, 1.2 and 2.3 have Lipschitz-completeness, so that they have the contraction property. Theorem 3.2 can be considered as a generalization of the Banach contraction principle under weaker completeness.

\section{The CONTRACTION PROPERTY AND ARCWISE-COMPLETENESS}

In this section, we consider the equivalence relation between completeness and the contraction principle in the sense of the equivalence metric.

Lemma 4.1 ([8]). Let $T$ operate on a metric space $(X, \rho)$. There exists a bounded metric $\sigma$ uniformly equivalent to $\rho$ on $X$ such that $T$ is a Banach contraction under $\sigma$ if, and only if, $T$ is uniformly continuous and

$$
\operatorname{diam}\left(T^{n} X\right) \rightarrow 0 .
$$

Theorem 4.2. Let $(X, d)$ be a metric space. If $X$ has the strong contraction property, then $X$ is arcwise-complete.

Proof. Let $\bar{X}$ be the completion of $X, g:(0,1] \mapsto X$ be a semi-closed arc and $\bar{x}=\lim _{s \rightarrow 0} g(s)$. Set $g(0)=\bar{x}$ and $X^{\prime}=X \cup\{\bar{x}\}$. Then $g$ continuously extends to a mapping on $[0,1]$.

Observe that $g(s) \rightarrow \bar{x}$ as $s \rightarrow 0$. For each number sequence $\left\{\epsilon_{k}\right\}_{k=1}^{\infty}$ with $1=\epsilon_{0}>\epsilon_{1}>\epsilon_{2}>\cdots$ and $\epsilon_{k} \rightarrow 0$ as $k \rightarrow \infty$, there exists a sequence $\left\{\delta_{k}\right\}_{k=1}^{\infty}$ such that $0 \leq \delta_{k+1}<\delta_{k} \leq \epsilon_{k}$ and

$$
d\left(g\left(t^{\prime}\right), g\left(t^{\prime \prime}\right)\right)<\epsilon_{k}, \quad \forall 0 \leq t^{\prime}, t^{\prime \prime}<\delta_{k} .
$$

Let $\bar{d}(x, y)=\frac{d(x, y)}{1+d(x, y)}$ and $h: X^{\prime} \rightarrow[0,1]$ be given by

$h(x)=\left\{\begin{array}{cl}\frac{\delta_{k+1}}{2^{k+1}} \cdot \frac{\bar{d}(x, \bar{x})-\epsilon_{k-1}}{\epsilon_{k}-\epsilon_{k-1}}+\frac{\delta_{k}}{2^{k}} \cdot \frac{\epsilon_{k}-\bar{d}(x, \bar{x})}{\epsilon_{k}-\epsilon_{k-1}}, & \epsilon_{k}<\bar{d}(x, \bar{x}) \leq \epsilon_{k-1}, k=1,2, \cdots ; \\ 0, & x=\bar{x} .\end{array}\right.$

Define $T: X^{\prime} \rightarrow X^{\prime}$ by

$$
T=g \circ h .
$$

It is easy to check that $T$ is uniformly continuous and $T \bar{x}=\bar{x}$. Furthermore, for each $x \in X$, we have

$$
|h(x)-0|=\left|\frac{\delta_{k+1}}{2^{k+1}} \cdot \frac{\bar{d}(x, \bar{x})-\epsilon_{k-1}}{\epsilon_{k}-\epsilon_{k-1}}+\frac{\delta_{k}}{2^{k}} \cdot \frac{\epsilon_{k}-\bar{d}(x, \bar{x})}{\epsilon_{k}-\epsilon_{k-1}}\right| \leq \frac{\delta_{k+1}}{2^{k+1}}+\frac{\delta_{k}}{2^{k}}<\delta_{k} .
$$


By (4.1),

$$
d(T x, \bar{x})=d(g(h(x)), g(0))<\epsilon_{k} .
$$

Since $\bar{d}(x, y) \leq d(x, y)$, without loss of generality, assume $\epsilon_{k^{\prime}+1}<\bar{d}(T x, \bar{x}) \leq \epsilon_{k^{\prime}}$ where $k^{\prime} \geq k$. Then

$|h(T x)-0|=\left|\frac{\delta_{k^{\prime}+2}}{2^{k^{\prime}+2}} \cdot \frac{\bar{d}(x, \bar{x})-\epsilon_{k^{\prime}}}{\epsilon_{k^{\prime}+1}-\epsilon_{k^{\prime}}}+\frac{\delta_{k^{\prime}+1}}{2^{k^{\prime}+1}} \cdot \frac{\epsilon_{k^{\prime}+1}-\bar{d}(x, \bar{x})}{\epsilon_{k^{\prime}+1}-\epsilon_{k^{\prime}}}\right| \leq \frac{\delta_{k^{\prime}+2}}{2^{k^{\prime}+2}}+\frac{\delta_{k^{\prime}+1}}{2^{k^{\prime}+1}}<\delta_{k+1}$.

By (4.1) again, this implies $d\left(T^{2} x, \bar{x}\right)=d(g(h(T x), g(0)))<\epsilon_{k+1}$. Continuing in this vein we get

$$
d\left(T^{n} x, \bar{x}\right)<\epsilon_{k+(n-1)} \leq \epsilon_{n}, \forall x \in X^{\prime} .
$$

Hence $\operatorname{diam}\left(T^{n}\left(X^{\prime}\right)\right) \rightarrow 0$ as $n \rightarrow \infty$ and the conditions of Lemma 4.1 are satisfied.

By Lemma 4.1, there is a metric $d^{*}$ uniformly equivalent to $d$ such that $T: X^{\prime} \mapsto$ $X^{\prime}$ is a Banach contraction mapping with respect to $d^{*}$. Thus $\bar{x}$ is the unique fixed point of $T$ in $X^{\prime}$. Finally observe that $T: X \rightarrow X$ and $T$ is also a metric contraction on $X$. The unique fixed point $\bar{x}$ of $T$ is in $X$ since $X$ has the strong contraction property.

Theorem 4.3. Let $(X, d)$ be a locally arcwise-connected metric space. If $X$ is arcwise-complete, then $X$ has the strong contraction property.

Proof. Let $d^{*}$ be a metric uniformly equivalent to $d, T: X \rightarrow X$ a metric contraction under $d^{*}$ with contraction constant $0 \leq h<1$. Note that $T$ is contractive and $d^{*}$ is uniformly equivalent to $d$. Select, as we may, $x_{0}$ in $X$ with $d\left(x_{0}, T x_{0}\right)<\delta$ and arc $g_{0}:[0,1] \rightarrow X$ which connect $x_{0}$ and $T x_{0}$. For each $k \in N$, define $g_{k}:[0,1] \rightarrow X$ by $g_{k}(s)=T^{k} g_{0}(s)$ for all $s \in[0,1]$. Then $g_{k}$ connects $x_{k}=T^{k}\left(x_{0}\right)$ and $x_{k+1}=T^{k+1}\left(x_{0}\right)$ for each $k \in N$. Now $\left\{T^{n} x_{0}\right\}$ is a Cauchy sequence under $d^{*}$, so that it is also a Cauchy sequence under $d$.

Define $g:(0,1] \mapsto X$ by

$$
g(s)=g_{k}\left(2^{k+1} s-1\right), \quad \forall \frac{1}{2^{k+1}}<s \leq \frac{1}{2^{k}}, \quad k=1,2, \cdots .
$$

Since $T$ is a metric contraction, it follows that $g$ is a semi-closed arc, $g(s) \rightarrow x^{*} \in X$ as $s \rightarrow 0$ and $\left\{T^{n} x_{0}\right\} \rightarrow x^{*}$. Finally, observe that $\left\{T^{n} x_{0}\right\}$ is a Cauchy sequence under $d$. It is easy to check that $x^{*}$ is the fixed point of $T$.

Theorem 4.3 can also be considered as a generalization of the Banach contraction principle under weaker completeness.

Combining Theorems 4.2 and 4.3, we have the following theorems.

Theorem 4.4. Let $X$ be a locally arcwise-connected metric space. Then $X$ is arcwise-complete if and only if $X$ has the strong contraction property.

Corollary 4.5. Let $X$ be an arcwise-connected metric space. Then $X$ is arcwisecomplete if and only if $X$ has the strong contraction property.

By Corollary 4.5, the subsets in Examples 1.1, 1.2 and 2.3 have the strong contraction property because, as we see, they have arcwise-completeness. 


\section{REFERENCES}

1. J. M. Borwein, Completeness and contraction principle, Proc. Amer. Math. Soc. 87 (1983), 246-250. MR0681829 (84a:54080)

2. J. R. Jachymski, Equivalence of some contractivity properties over metrical structures, Proc. Amer. Math. Soc. 125(1997),2327-2335. MR1389524|(97j:54047)

3. J. R. Jachymski, Short proof of the converse to the contraction principle and some related results, Topol. Methods Nonlinear Anal. 15(2000),179-186. MR:1786260 (2001h:54071)

4. L. Janos, A converse of Banach's contraction theorem, Proc. Amer. Math. Soc. 18(1968),287289. MR0208589 (34:8398)

5. L. Janos, On pseudo-complete spaces, Notices Amer. Math. Soc. 18(1971), 97-163.

6. L. Janos, The Banach contraction mapping principle and cohomology, Comment. Math. Univ. Carolinae, 43:3(2000),605-610. MR 1795089 (2001m:54039)

7. W. A. Kirk, Caristi's fixed point theorem and metric convexity, Colloq. Math. 36(1976), 81-86. MR0436111 (55:9061)

8. S. Leader, A topological characterization of Banach contractions, Pacific J. Math. 69:2(1977),461-466. MR0436093 (55:9044)

9. F. Sullivan, A characterization of complete metric spaces, Proc. Amer. Math. Soc. 83(1981),345-346. MR0624927 (83b:54036)

Department of Mathematics, Guizhou University, Guiyang, Guizhou 550025, People's Republic of China

E-mail address: shwxiang@vip.163.com 